\title{
Cognitive and Psychological Outcomes in Younger vs. Older Children with Subtentorial/Supratentorial Ependymoma after Radiation Therapy
}

\author{
Geraldina Poggi ${ }^{1}$, Annarita Adduci ${ }^{1}$, Lorenza Gandola ${ }^{2}$, Susanna Galbiati ${ }^{1}$, \\ Alessandro Degrate ${ }^{1}$, Elena Sironi ${ }^{1}$, Sandra Strazzer ${ }^{1}$, Maura Massimino ${ }^{2}$ \\ ${ }^{1}$ Scientific Institute E. Medea, Bosisio Parini, Italy \\ ${ }^{2}$ National Tumor Institute, Milan, Italy \\ E-mail: geraldina.poggi@bp.Inf.it \\ Received March 28, 2011; revised April 20, 2011; accepted May 12, 2011
}

\begin{abstract}
Purpose: to investigate cognitive and psychological problems in children treated for intracranial ependymoma, the evolution of these disorders over time and the role of age at radiotherapy and tumor site, in their onset and persistence. Methods and Materials: 23 patients received a complete evaluation; some of them underwent follow-ups. The clinical data collected included sex, age (at diagnosis, assessment and tumor treatment, thus dividing patients into two cohorts: younger or older than 5 years), site (supratentorial vs. subtentorial), the presence of hydrocephalus, neurological examination, tumor treatment. All the patients received an age-appropriate cognitive and psychological evaluation. Results: The mean cognitive level was within the norm, with lower scores on the Performance Intelligence Quotient (PIQ). The psychological assessment revealed Internalizing problems and impaired independence. Children older than 5 years had a lower Intelligence Quotient (IQ) than the younger children, both at the initial evaluation and at follow-ups. Initially, the supratentorial group appeared to be less impaired than the subtentorial group but then exhibited a progressive decline in the IQ. In the subtentorial group, the children with an IQ within the normal range remained stable at the follow-up, while the children with below-norm scores at the initial evaluation showed deterioration over time. Conclusions: Tumor site seems to affect the cognitive outcome to a greater extent than age at radiotherapy.
\end{abstract}

Keywords: Cognitive Outcome, Ependymoma, Children, Radiotherapy

\section{Introduction}

Studies on the cognitive outcome of patients with ependymoma are limited in number and are generally part of larger studies where ependymomas are compared with other posterior fossa $(\mathrm{PF})$ tumors such as medulloblastomas and astrocytomas [1-4].

The most recent studies on cognitive and neuropsychological outcomes show that patients treated for ependymoma do not present with severe cognitive impairment as compared to other brain tumors, even though many of them have neuropsychological problems (affecting memory, attention, language and executive functions) which often impair formal learning $[5,6]$.

As for all brain tumors, age at diagnosis and the quality of surgery and radiotherapy are considered to be the most important predictive factors for the onset of cognitive and neuropsychological problems which have a great impact on long-term survival [7-11].

The impact of radiation therapy was explored in Merchant's studies which showed how Conformal Radiation Therapy (CRT) pointed out that supratentorial tumor location, multiple surgery, male sex, longer symptomatic interval, pre-CRT chemotherapy, endocrine deficiencies, hydrocephalus, and young age at CRT were predictive factors for academic problems [12-17].

In a recent work on 23 patients treated with local posterior fossa irradiation who received one or more consecutive neuropsychological evaluations, von Hoff found a moderate impairment of intellectual functions and identified early disease manifestation and treatment and postoperative neurological deficits as the main risk factors 
for the onset of cognitive problems. This study also emphasized that the neuropsychological difficulties can vary in type and that no distinctive pattern of impairment can be identified, unlike in other tumors [18].

The present study thus set out to:

1) Define the influence of age at radiotherapy as a predictive factor by comparing two subgroups according to age at radiotherapy (children younger than 5 years and children older than 5 years).

2) Define the influence of tumor site as a predictive factor by comparing two subgroups according to supraor subtentorial site.

3) Define the cognitive and psychological evolution of the whole group's cognitive profile over time.

\section{Material and Methods}

\section{Patients}

This study included the newly diagnosed patients treated for ependymoma at the Pediatric Oncology Department of the National Institute for Tumors of Milan in the time period between 1993 and 2005, according to two consecutive national AIEOP (Italian Association of Pediatric Hematology and Oncology) protocols.

In the 1994-2001 period, the patients with complete tumor excision received hyperfractionated radiotherapy (1.1 Gy twice a day) to the tumor bed plus $1-2 \mathrm{~cm}$ margins up to a total dose of $70.4 \mathrm{~Gy}$. When the residual tumor was documented after surgery, the patients first received 4 chemotherapy courses with vincristine, etoposide and cyclophosphamide (VEC) plus/minus secondlook surgery, followed by the same radiotherapy schedule (protocol 1) [19]. After 2001, the children with complete tumor excision and grade 2 revised histology received conformal radiation with conventional fractionation of $1.8 \mathrm{~Gy} / \mathrm{d}$ up to a total dose of $59.4 \mathrm{~Gy}$. The children with complete tumor excision and grade 3 revised histology received $4 \mathrm{VEC}$ courses after radiotherapy. In the presence of a residual tumor, of any grade, VEC was prescribed before radiotherapy to facilitate second-look tumor removal. In the event of there still being a tumor after the second-look surgery, radiotherapy was completed with a boost delivered in two daily fractions of 4 Gy (protocol 2). Exclusion criteria included a history of previous brain lesions (congenital or acquired), pre-existing acute or chronic serious illness and psychomotor delay, as reported by the parents. All the patients received at least one complete cognitive evaluation.

A total of 23 patients were included in the study. After the initial assessment, some patients underwent further evaluation: 13 patients were assessed only once, 10 patients twice, 6 patients 3 times and 4 patients 4 times.

The progressive reduction in number in the patients participating in the follow-ups is due to that fact that about half of the 23 patients initially evaluated lived at a significant distance from their oncological centers and our Institute to which they had been referred for a multidisciplinary clinical and functional evaluation at the end of tumor treatment. These patients were then referred to more easily accessible centers and did not undergo later follow-ups.

All the participants gave their consent. The following clinical and medical data were collected for all of them: sex, age at diagnosis, age at assessment, age at tumor treatment, time between diagnosis and assessment, histological diagnosis, tumor site (supratentorial vs. subtentorial), hydrocephalus, neurological examination and type of tumor treatment (neurosurgery, chemotherapy and radiotherapy).

\section{Methods}

\section{Cognitive/Neuropsychological and Psychological Evaluation}

All the patients received an age-appropriate clinical cognitive and psychological evaluation.

The cognitive assessment was performed using the Wechsler Intelligence Scales-WPPSI-R, WISC-R [20, 21]. Attention, memory, executive functions, were also evaluated by age-appropriate tests (see Table 1).

The use of different tests did not allow for a quantitative analysis of the neuropsychological evaluation: for that reason, in this study, the only variable considered is the presence or the absence of specific problems in those investigated areas (attention, memory, executive functions). Thus, a qualitative analysis was performed.

The psychological evaluation protocol was geared to assess several aspects of psychosocial functioning. The Child Behavior Checklist (CBCL) [22] and the Vineland Adaptive Behavior Scales-Expanded Form (VABS) [23] were administered.

\section{Statistical Analysis}

Statistical analysis was conducted using SPSS software.

Table 1. Neuropsychological domain and test of measurement.

\begin{tabular}{cc}
\hline \multicolumn{2}{c}{ Neuropsychological domain and test of measurement } \\
\hline \multirow{2}{*}{ Attention } & - Conners' Kiddie Continuous Performance \\
& Test (K-CPT) \\
& - Continuous Performance Test II (CPT II) \\
Memory & - Rey Figure - memory - \\
& - Digit Span \\
Executive Functions & - Wisconsin Card Sorting Test (WCST) \\
\hline
\end{tabular}


Data are presented as means and standard deviations (SDs) for quantitative variables, and as frequencies and percentages for qualitative variables. The scores of some neuropsychological tests were transformed to Z-Scores. A $Z$-score $\geq 2$ was considered to be pathological.

With regard to the associations between cognitivepsychological problems and site and age at radiotherapy, the findings were compared by means of an Analysis of Variance (ANOVA): multiple comparisons between subgroups of patients were performed based on age at radiotherapy, site. A matched-pair $t$-test sample compared the different evaluation outcome within the whole group. A $p$ value $<0.05$ (two-tailed) was considered to be significant.

\section{Results}

\section{Sample Description at $\mathbf{1}^{\text {st }}$ Evaluation}

Table 2 shows the demographic and clinical characteristics of the whole group ( 23 patients) at the first evaluation.

As shown in Table 2, most of the patients had been treated for a subtentorial ependymoma. Of these patients, $26 \%$ had developed hydrocephalus at tumor onset. Most of the patients were treated according to Protocol 2. The most frequently reported outcomes include motor problems, while sensory problems were found in a small number of patients. No patient presented with epilepsy.

With regard to the cognitive evaluation, at the initial assessment the group presented with mean Full Intelligence Quotient (FIQ), Verbal Intelligence Quotient (VIQ) and Performance Intelligente Quotient (PIQ) scores within the norm, although four $(17.4 \%)$ patients had an FIQ below the norm.

In the neuropsychological test, $9(39.1 \%)$ patients showed attention problems, $5(21.7 \%)$ patients had memory problems and $9(39.1 \%)$ patients had executive function deficits.

With regard to the psychological evaluation, the most frequently reported problem on the CBCL is Internalizing: $6(26 \%)$ patients scored above the norm on the "Internalizing" scale. The VABS shows a greater impairment in the motor domain and independence. Table 2 also shows the mean scores obtained by the patients in the psychological tests.

\section{- Age at Radiotherapy}

When radiotherapy was administered, 10 (43.4\%) children were aged $<5$ years and $13(56.5 \%)$ children were aged $>5$ years. When looking at the relationship between age at radiotherapy and cognitive outcome, there is a marked, yet not statistically significant, difference in IQ between the two groups: the older children have a lower mean IQ than the younger children. The difference in
FIQ is close to significance ( $p=0.07)$. With regard to the relationship between age at radiotherapy and psychological outcomes, the mean CBCL scale scores of the two groups are similar. In contrast, the VABS scores show a greater impairment in the older group, but this difference is not significant.

\section{- Tumor Site}

With regard to the supra-/subtentorial distinction, the initial cognitive evaluation revealed a greater impairment in the subtentorial group, with VIQ, PIQ and FIQ scores of $99.25,91.58$ and 96.6 in the subtentorial group and 102, 105.6 and 105 in the supratentorial group. The greater impairment of the subtentorial group appears to be confirmed by the VABS scores evidencing an impairment in independence and social skills. In contrast, the CBCL scores do not show any relevant differences.

\section{Evolution over Time}

To study the cognitive, neuropsychological and psychological outcomes evolution over time we opted for a qualitative analysis because of the attrition in the number of patient participating to this research; we performed a qualitative evaluation of each individual case and gave a description of the progress over time in the 11 cases undergoing follow-ups (see Table 3).

With regard to the initial evaluation, most cases were within the norm: 20 patients had an IQ of over 75, but three patients had a score below the norm; 11 patients showed neuropsychological difficulties, but only 7 patients showed a deficit in all neuropsychological areas. 10 patients had psychosocial problems.

With regard to progress over time, 6 patients remained stable or improved, and in all 6 cases these patients had a history of subtentorial tumors. However, we noticed a decline in the cognitive level of 5 patients at follow-up. Of the latter, almost all (4 patients) had a supratentorial tumor. The $5^{\text {th }}$ patient had a subtentorial tumor. Unlike the other cases who had average scores at the initial evaluation, he already had an impaired cognitive level attributable to neurological lesions and sensory impairments which appeared after hydrocephalus at the tumor onset and neurosurgery.

Of the 5 patients whose condition worsened, two belonged to the group of patients aged less than 5 years at radiotherapy and three belonged to the group of patients aged more than 5 years at radiotherapy.

With regard to the site, we observed a tendency towards deterioration in patients with a supratentorial tumor and a tendency towards stability-rather than an improvement in those with a subtentorial tumor, regardless of age. The qualitative analysis revealed that site had a greater influence than age on the progress of the cogni- 
Table 2. Sample description at 1st evaluation.

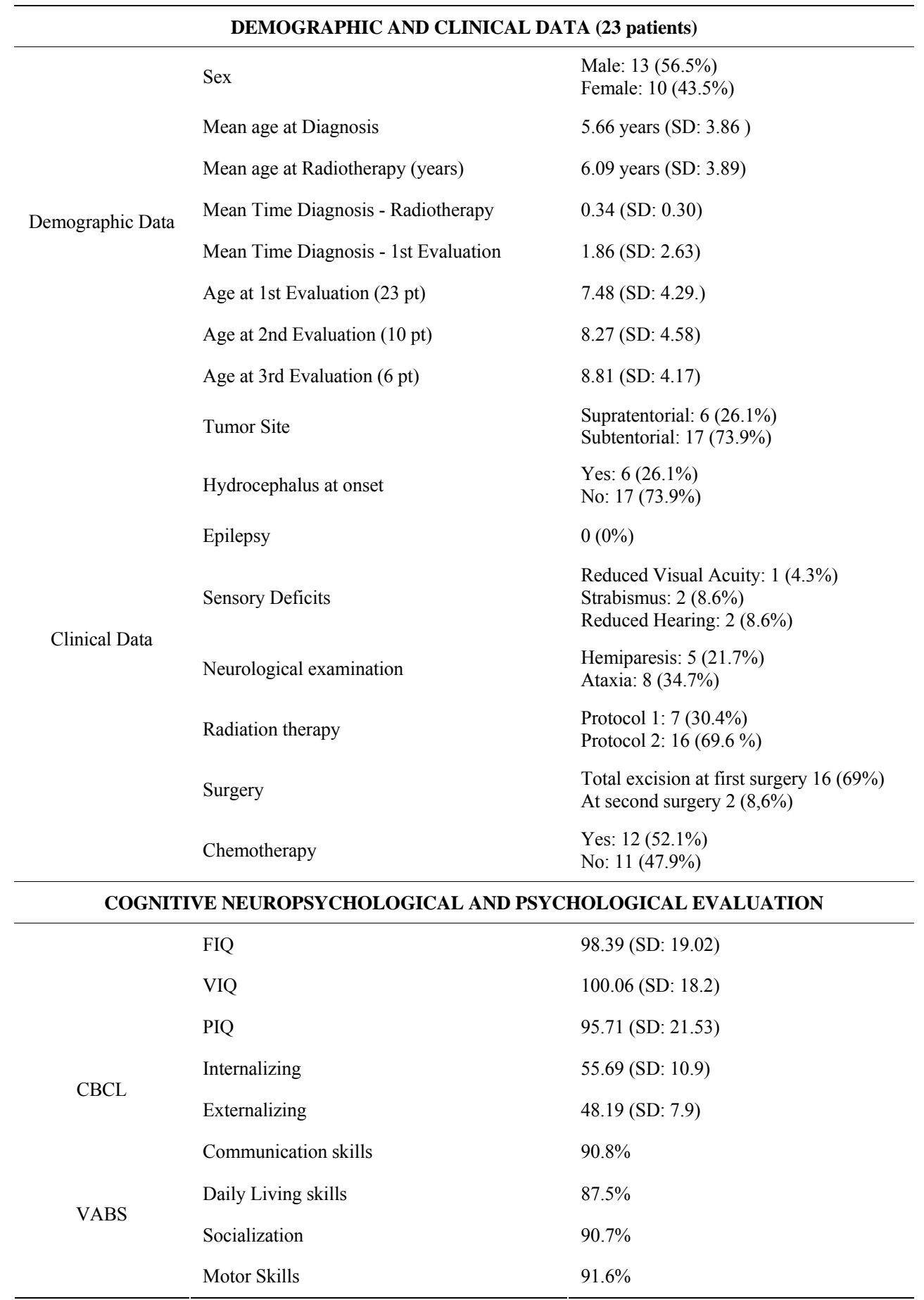

tive situation over time.

\section{Discussion}

We studied the clinical and cognitive, neuropsychological and psychological profiles of patients in order to reveal how age at radiotherapy and tumor site have influ- ence in defining this profile, describing how this evolve over time.

As compared to other populations, our sample fares better from a clinical point of view in that our patients did not exhibit severe motor or cognitive impairments. Their outcomes are definitely more favorable than that of patients who received whole brain irradiation [1-3]. The 
Table 3. Qualitative analysis.

\begin{tabular}{|c|c|c|c|c|c|c|c|c|c|c|c|}
\hline PT & $\begin{array}{c}\text { Age at } \\
\text { Diagnosis }\end{array}$ & $\begin{array}{l}\text { Age at } \\
\text { RT }\end{array}$ & $\begin{array}{c}\text { Age at } \\
\text { First } \\
\text { Evaluation }\end{array}$ & $\begin{array}{c}\text { Age } \\
\text { Group }\end{array}$ & $\begin{array}{l}\text { Tumor } \\
\text { Site }\end{array}$ & $\begin{array}{l}\text { IQ at First } \\
\text { Evaluation }\end{array}$ & $\begin{array}{l}\text { Cognitive } \\
\text { Evolution } \\
\text { over time }\end{array}$ & $\begin{array}{l}\text { Attention } \\
\text { Problems }\end{array}$ & $\begin{array}{l}\text { Memory } \\
\text { Problems }\end{array}$ & $\begin{array}{l}\text { Executive } \\
\text { Function } \\
\text { Problems }\end{array}$ & $\begin{array}{c}\text { Psycho-Social } \\
\text { Problems }\end{array}$ \\
\hline 1 & 26 & 29 & 36 & $<5$ & Supra & 110 & Deterioration & No & l & l & Yes \\
\hline 2 & 37 & 45 & 51 & $<5$ & Supra & 123 & Deterioration & No & No & I & No \\
\hline 3 & 17 & 20 & 28 & $<5$ & Sub & 112 & // & l & l & I & Yes \\
\hline 4 & 12 & 14 & 24 & $<5$ & Sub & 102 & // & I & l & l & No \\
\hline 5 & 19 & 22 & 30 & $<5$ & Sub & 98 & // & / & l & l & No \\
\hline 6 & 27 & 39 & 45 & $<5$ & Sub & 91 & // & / & l & / & Yes \\
\hline 7 & 37 & 37 & 39 & $<5$ & Sub & 101 & Stable & I & I & l & Yes \\
\hline 8 & 42 & 47 & 48 & $<5$ & Sub & 134 & Improvement & No & No & No & No \\
\hline 9 & 48 & 56 & 178 & $<5$ & Sub & 67 & // & Yes & Yes & Yes & Yes \\
\hline 10 & 50 & 55 & 65 & $<5$ & Sub & 117 & // & No & No & l & No \\
\hline 11 & 54 & 56 & 58 & $<5$ & Sub & 113 & Improvement & No & No & No & No \\
\hline 12 & 60 & 63 & 68 & $>5$ & Supra & 98 & Deterioration & Yes & Yes & Yes & Yes \\
\hline 13 & 65 & 67 & 72 & $>5$ & Supra & 107 & // & Yes & No & No & No \\
\hline 14 & 103 & 105 & 131 & $>5$ & Supra & 95 & // & No & No & No & No \\
\hline 15 & 170 & 176 & 187 & $>5$ & Supra & 97 & Deterioration & Yes & Yes & Yes & Yes \\
\hline 16 & 60 & 64 & 73 & $>5$ & Sub & 70 & Deterioration & Yes & Yes & Yes & Yes \\
\hline 17 & 63 & 66 & 144 & $>5$ & Sub & 86 & // & Yes & Yes & Yes & No \\
\hline 18 & 68 & 82 & 92 & $>5$ & Sub & 101 & Stable & Yes & Yes & Yes & No \\
\hline 19 & 72 & 73 & 156 & $>5$ & Sub & 93 & // & No & No & Yes & Yes \\
\hline 20 & 103 & 105 & 112 & $>5$ & Sub & 54 & // & Yes & Yes & Yes & Yes \\
\hline 21 & 117 & 119 & 118 & $>5$ & Sub & 90 & Stable & Yes & No & Yes & No \\
\hline 22 & 132 & 134 & 150 & $>5$ & Sub & 115 & Stable & No & No & No & No \\
\hline 23 & 180 & 182 & 185 & $>5$ & Sub & 105 & /I & Yes & No & No & No \\
\hline
\end{tabular}

LEGEND: $\mathrm{RT}=$ radiotherapy; SUPRA = supratentorial site; $\mathrm{SUB}=$ subtentorial site.

most frequently reported motor impairments included hemiparesis and ataxia in percentages similar to those reported in the literature $[1,3,17]$. They are almost totally attributable to a preoperative lesion or immediate postoperative lesion.

At the cognitive and neuropsychological levels, the mean scores of the whole sample are within the norm. The same holds true for the PIQ scores which were lower than the VIQ scores. The difference between PIQ and VIQ is consistent with the literature.

On the whole, the picture was stable from a neuropsychological point of view as well; at the first evaluation, 4 of the 7 patients with significant neuropsy-chological problems had scored below or at the lower limits of the norm: these were patients with pre- or periopera- tive damage. 3 patients had neuropsychological difficulties which were associated with a subsequent deterioration of the cognitive level rather than an initial impairment. The neuropsychological problems did not appear to be necessarily associated to an impairment of the cognitive level. This is in line with recent reports of core problems (attention and memory problems) affecting academic performance, even in the presence of non-pathological cognitive levels [24,25].

It is assumed that disorders of attention, memory and executive functions - the functions underlying learning - impair the normal functional development process and, in so doing, affect contextual and school learning [26].

It is therefore necessary to perform repeat follow-ups over time as the presence of disorders affecting the basic 
neuropsychological functions could adversely influence later cognitive development and planning, organization, categorization and cognitive flexibility, thus interfering with learning and interpersonal skills [24,25].

While investigating the outcome, relevant findings emerged when we divided the patients into groups according to age at radiotherapy and tumor site (supra- vs. subtentorial). With regard to age we found a greater cognitive impairment in the older patients at the initial evaluation than at the later follow-ups. Such a difference was seen in both performance and verbal tests.

With regard to tumor site, we found a greater initial impairment in patients with a subtentorial tumor, while patients with a supratentorial tumor showed a more favorable initial pattern which however deteriorated progressively at the follow-ups, both in performance and verbal tests.

In contrast, the subtentorial group remained stable or improved during time, except for the patient with scores below the limits of the norm at the initial cognitive evaluation who later exhibited a deterioration.

When looking at the most significant variables being studied (age, site), the limited sample size and heterogeneity does not enable us to draw representative conclusions on the differences in the clinical picture. However, it can be stated that the younger patients who were treated with radiotherapy do not appear to have a cognitive impairment. Only one out of 11 patients showed a generalized cognitive impairment, with an association between the below-the-norm IQ and neuropsychological psychological problems.

When interpreting this finding, it should be kept in mind that only 6/11 patients in the younger group underwent a neuropsychological evaluation (attention, memory and executive functions) because the remaining children were too young for these tests.

The most frequently observed psychological problems are Internalizing problems. The result of the psychologycal evaluation which was performed on our sample is in line with previous studies, which found the same kind of disorders affecting this population of patients.

In conclusion, we can safely state that, in our sample, tumor site seems to affect the cognitive outcome to a greater extent than age at radiotherapy.

This emphasizes the need for similar studies on larger samples which can provide a better insight into the multiplicity of cognitive, neuropsychological and psycho-logical profiles and enable a more detailed quantitative analysis to be made, together with more generalizable conclusions. Such studies should be long-term, as longitudinal observations enable better monitoring of the cognitive, neuropsychological and psychological problems and their impact on later cognitive development.

\section{Acknowledgements}

We gratefully acknowledge the financial support of AIRC (Associazione Italiana per la ricerca contro il cancro).

\section{References}

[1] L. Levisohn, A. Cronin-Golomb and J. D. Schmahmann, "Neuropsychological Consequences of Cerebellar Tumour Resection in Children: Cerebellar Cognitive Affective Syndrome in a Paediatric Population," Brain, Vol. 123, No. 5, 2000, pp. 1041-1050. doi:10.1093/brain/123.5.1041

[2] D. W. Weebe, M. D. Ris, F. D. Armstrong, et al., "Cognitive and Adaptive Outcome in Low-Grade Pediatric Cerebellar Astrocytomas: Evidence of Diminished Cognitive and Adaptive Functioning in National Collaborative Research Studies (CCG 9891/POG 9130)," Journal of Clinical Oncology, Vol. 23, No. 22, 2005, pp. 5198- 5202. doi:10.1200/JCO.2005.06.117

[3] R. Stargatt, J. V. Rosenfeld, W. Maixner, et al., "Multiple Factors Contribute to Neuropsychological Outcome in Children with Posterior Fossa Tumors," Developmental Neuropsychology, Vol. 32, 2007, pp. 729-748. doi.org/10.1080/87565640701376151

[4] R. K. Mulhern, S. L. Palmer, T. E. Merchant, et al., "Neurocognitive Consequences of Risk-Adapted Therapy for Childhood Medulloblastoma," Journal of Clinical Oncology, Vol. 23, No. 24, 2005, pp. 5511-5519. doi.org/10.1200/JCO.2005.00.703

[5] E. Hoppe-Hirsch, L. Brunet, F. Laroussinie, et al., "Intellectual Outcome in Children with Malignant Tumors of the Posterior Fossa: Influence of the Field of Irradiation and Quality of Surgery," Child's Nervous System, Vol. 11, No. 6, 1995, pp. 340-346. doi:10.1007/BF00301666

[6] J. Grill, V. K. Renaux, C. Bulteau, et al., "Long-Term Intellectual Outcome in Children with Posterior Fossa Tumors according to Radiation Doses and Volumes," International Journal of Radiation Oncology, Vol. 45, No. 1, 1999, pp. 137-145. doi:10.1016/S0360-3016(99)00177-7

[7] J. Grill, D. Viguier, V. Kieffer, et al., "Critical Risk Factors for Intellectual Impairment in Children with Posterior Fossa Tumors: The Role of Cerebellar Damage," Journal of Neurosurgery, Vol. 101, No. 2, 2004, 152-158.

[8] E. N. Kiehna, R. K. Mulhern, C. Li, et al., "Changes in Attentional Performance of Children and Young Adults with Localized Primary Brain Tumors after Conformal Radiation Therapy," Journal of Clinical Oncology, Vol. 24, No. 33, 2006, pp. 5283-5290. doi:10.1200/JCO.2005.03.8547

[9] V. Kieffer-Renaux, D. Viguier, M. A. Raquin, et al., "Therapeutic Schedules Influence the Pattern of Intellectual Decline after Irradiation of Posterior Fossa Tumors," Pediatr Blood Cancer, Vol. 45, No. 6, 2005, pp. 814-819. doi: $10.1002 /$ pbc. 20329 
[10] E. Seaver, R. Gever, S. Sulzbacher, et al., "Psychosocial Adjustment in Long-Term Survivors of Childhood Medulloblastoma and Ependymoma Treated with Craniospinal Irradiation," Pediatr Neurosurg, Vol. 20, No. 4, 1994, pp. 248-253. doi:10.1159/000120798

[11] J Grill M-C. Le Deley, D. Gambarelli, et al., "Postoperative Chemotherapy without Irradiation for Ependymoma in Children under 5 Years of Age: A Multicenter Trail of the French Society of Pediatric Oncology," Journal of Clinical Oncology, Vol. 19, 2001, pp. 1288-1296.

[12] T. E. Merchant and M. Fouladi, "Ependymoma: New Therapeutic Approaches Including Radiation and Chemotherapy," Journal of Neuro-Oncology, Vol. 75, No. 3, 2005, 287-299. doi:10.1007/s11060-005-6753-9

[13] T. E. Merchant, E. N. Kiehna, C. Li, et al., "Radiation Dosimetry Predicts IQ after Conformal Radiation Therapy in Pediatric Patients with Localized Ependymoma," International Journal of Radiation Oncology, Vol. 63, No. 5, 2005, pp. 1546-1554. doi:10.1016/j.ijrobp.2005.05.028

[14] T. E. Merchant, C. Li, X. Xiong, et al., "Conformal Radiotherapy after Surgery for Paediatric Ependymoma: A Prospective Study," The Lancet Oncology, Vol. 10, No. 3, 2009, 258-266. doi:10.1016/S1470-2045(08)70342-5

[15] H. M. Conklin, C. Li, X. Xiong, et al., "Predicting Change in Academic Abilities after Conformal Radiation Therapy for Localized Ependymoma," Journal of Clinical Oncology, Vol. 26, No. 24, 2008, 3965-3970. doi:10.1200/JCO.2007.15.9970

[16] T. E. Merchant, R. K. Mulhern, M. J. Krasin et al., "Preliminary Results from A Phase II Trial of Conformal Radiation Therapy and Evaluation of Radiation-Related CNS Effects for Pediatric Patients with Localized Ependymoma," Journal of Clinical Oncology, Vol. 22, No. 15, 2004, pp. 3156-3162. doi:10.1200/JCO.2004.11.142

[17] T. E. Merchant, H. Lee, J. Zhu, et al., "The Effects of Hydrocephalus on Intelligence Quotient in Children with Localized Infratentorial Ependymoma before and after Radiation Therapy," Journal of Neurosurgery, Vol. 101, 2004, pp. 159-168.

[18] K. Von Hoff, V. Kieffer, J.-L. Habrand, et al., "Impairment of Intellectual Functions after Surgery and Posterior Fossa Irradiation in Children with Ependymoma Is Re- lated to Age and Neurologic Complications," BMC Cancer, 2008, Vol. 8, p. 15. doi:10.1186/1471-2407-8-15

[19] M. Massimino, L. Gandola, F. Giangaspero, et al., for the AIEOP Pediatric Neuro-Oncology Group, "Hyperfractionated Radiotherapy and Chemotherapy for Childhood Ependymoma: Final Results of the First Prospective AIEOP (Associazione Italiana di Ematologia-Oncologia Pediatrica) Study," International Journal of Radiation Oncology, Vol. 58, No. 5, 2004, pp. 1336-1345. doi:10.1016/j.ijrobp.2003.08.030

[20] D. Wechsler, "Manual for the Wechsler preschool and primary scale of Intelligence-Revised," The Psychological Corporation, San Antonio, 1989.

[21] D. Wechsler, "Wechsler Intelligence Scale for Children," 3rd Edition, The Psychological Corporation, San Antonio 1989.

[22] T. M. Achenbach and C. Edelbrock, "The Child Behavior Checklist and Revised Child Behavior Profile," Thomas J. Achenbach, Burlington, 1983.

[23] S. Sparrow, D. A. Balla and D. V. Cicchetti, "Vineland Adaptive Behavior Scale," Circe Pines (MN), American Guidance Service, 1984.

[24] S. L. Palmer, "Neurodevelopmental Impact on Children Treated for Medulloblastoma: A Review and Proposed Conceptual Model," Developmental Disabilities Research Reviews, Vol. 14, No. 3, 2008, pp. 203-210. doi: $10.1002 /$ ddrr. 32

[25] D. J. Mabbot, L.Penkman, A. Witol, et al., "Core Neurocognitive Functions in Children Treated for Posterior Fossa Tumors," Neuropsychology, Vol. 22, No. 2, 2008, pp. 159-168. doi:10.1037/0894-4105.22.2.159

[26] M.-E. Briere, J. G. Scott, R. Y. McNall-Knapp, et al., "Cognitive Outcome in Pediatric Brain Tumor Survivors: Delayed Attention Deficit at Long-Term Follow-up," Pediatr Blood Cancer, Vol. 50, No. 2, 2008, 337-340. doi: $10.1002 / \mathrm{pbc} .21223$

[27] M. Benesch, K. Spiegl, A. Winter, et al., "A Scoring System to Quantify Late Effects in Children after Treatment for Medulloblastoma/Ependymoma and Its Correlation with Quality of Life and Neurocognitive Functioning", Child's Nervous System, Vol. 25, No. 2, 2009, pp. 173-181. doi:10.1007/s00381-008-0742-1 